
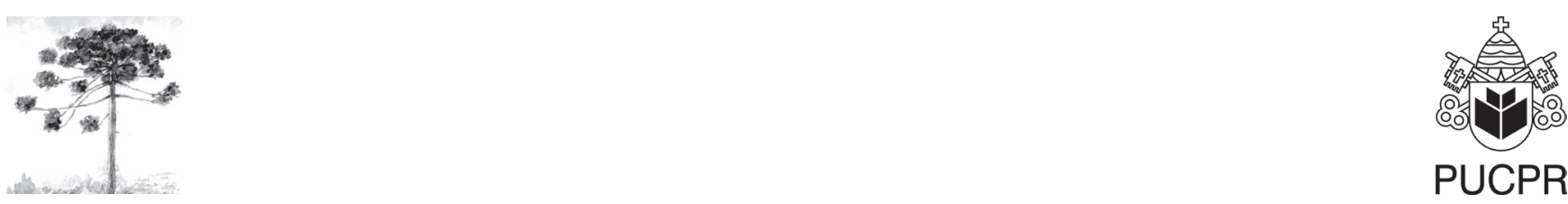

PUCPR

\title{
TENDOPARASITOSES EM PUMA (Puma concolor) NA REGIÃO SUL DO BRASIL
}

\author{
Endoparasitosis in congar (Puma concolor) in the Southern Brazil
}

\author{
Vinícius da Rosa Fanfa ${ }^{[\mathrm{a}]}$, Matheus Hilliard Farret ${ }^{[\mathrm{a}]}$, Aleksandro Schafer da Silva ${ }^{[\mathrm{b}]}$, \\ Silvia Gonzalez Monteiro ${ }^{[c]}$
}

[a] Curso de Medicina Veterinária da Universidade Federal de Santa Maria (UFSM), Santa Maria, RS - Brasil, e-mail: viniciusfanfa@hotmail.com

[b] Pós-Graduação em Medicina Veterinária da Universidade Federal de Santa Maria (UFSM), Santa Maria, RS - Brasil, e-mail: aleksandro_ss@yahoo.com.br

[c] Departamento de Microbiologia e Parasitologia da Universidade Federal de Santa Maria (UFSM), Santa Maria, RS - Brasil, e-mail: sgmonteiro@uol.com.br

\section{Resumo}

O puma (Puma concolor) é um felídeo encontrado em diversas zonas ecológicas do Brasil e Américas. Em pumas já foi reportado parasitismo por carrapatos ixodidae e endoparasitos de diferentes gêneros e espécies. Porém, pesquisas envolvendo a fauna silvestre brasileira ainda são poucas. Portanto, este estudo visou a avaliar o parasitismo gastrintestinal em $P$. concolor mantido em cativeiro na região central do Rio Grande do Sul. Foram analisadas fezes de um puma adulto através dos métodos exame direto, centrífugo flutuação com sulfato de zinco e kinyon para pesquisa de parasitos. Nas amostras constatou-se a presença de infecção mista por oocistos de Cryptosporidium sp. e ovos Trichuris sp., além de oocistos de coccídeos sugestivos de Toxoplasma gondii ou Besnoitia sp. Com base nos resultados, pode-se concluir que o puma é um hospedeiro em potencial desses parasitos, apresentando quadro clínico assintomático. No entanto, cabe pesquisar o verdadeiro papel desse felídeo no ambiente relacionado à disseminação desses parasitos.

Palavras-chave: Cryptosporidium. Toxoplasma gondii. Besnoitia. Trichuris.

\section{Abstract}

Cougar (Puma concolor) is a feline found in various ecological zones in Brazil and Americas. In cougar has been reported parasitism by ticks ixodidae and endoparasites of different genera and 
species. However, researches involving the Brazilian wild fauna are still few. Therefore, this study aimed to evaluate the gastrointestinal parasitism in $\mathrm{P}$. concolor kept in captivity in the central region of Rio Grande do Sul state. Fecal samples of an adult cougar were analyzed by the direct smear method, centrifugal flotation technique with zinc sulfate and kinyon staining method. Mixed infection by oocysts of Cryptosporidium sp. and eggs of Trichuris $s p$. were observed in the samples, besides oocysts of coccidea suggestive of Toxoplasma gondii or Besnoitia sp. Based on the results, it can be concluded that the cougar is a potential host of these parasites, presenting with asymptomatic. However, the true role of the feline in environment related to the spread of these parasites has to be studied.

Keywords: Cryptosporidium. Toxoplasma gondii. Besnoitia. Trichuris.

\section{INTRODUÇÃO}

O puma (Puma concolor), também conhecido como leão da montanha, onça parda ou suçuarana é um felídeo encontrado em diversas zonas ecológicas do Brasil e das Américas. Pode habitar os mais diversos tipos de ecossistemas, como desertos, savanas, estepes, caatinga, cerrado, Pantanal, Floresta Amazônica e mata atlântica. São felinos exclusivamente carnívoros, com dieta à base de mamíferos, aves e répteis. Destaca-se por sua visão e audição bem desenvolvidas, que o tornam um grande predador (1-3).

A infecção por protozoários e helmintos em animais ocorre geralmente pela ingestão da forma infectante presente nos alimentos ou água (4). Em pumas a literatura reporta parasitismo por carrapatos ixodidae e endoparasitos como Toxocara sp., Capillaria sp., Trichuris sp., Ancylostoma sp., Cryptosporidium sp., Spirometra sp., Toxoplasma gondii, Sarcocystis sp., Taenia omissa, Isospora felis, Alaria marcianna, Cylicospirura sp., Lagochilascaris sp. (5-12).

Com a crescente preocupação relativa à atuação de animais silvestres como portadores e vinculadores de zoonoses em saúde pública, considerou-se oportuno relatar a ocorrência de parasitos gastrintestinais em $P$. concolor no sul do País.

Foram coletadas amostras de fezes de um puma adulto mantido em um criadouro conservacionista em Santa Maria no Rio Grande do Sul, Brasil. A amostra foi armazenada em frascos de polietileno, em temperatura de $13^{\circ} \mathrm{C}$ por 24 horas. Essa foi processada através das técnicas de centrífugo flutuação, exame direto e kinyon para pesquisa de parasitos. Os oocistos de coccídeos foram mantidos em temperatura e umidade controlada $\left(27^{\circ} \mathrm{C}\right.$ e $80 \%$ UR) por cinco dias, para esporulação a fim de identificação do gênero (13).
No exame direto e centrífugo flutuação observou-se a presença de ovos de Trichuris sp., oocistos de Cryptosporidium sp. e outro coccídeo. $\mathrm{Na}$ coloração de Kinyon confirmou-se a presença de Cryptosporidium sp. Após esporulação dos oocistos, identificaram-se os mesmos pela morfologia e tamanho como Toxoplasma gondii ou Besnoitia sp. Essa diferenciação não é possível devido à semelhança entre os dois, sendo necessárias técnicas mais especificas (PCR). A infecção foi considerada leve para todos os parasitos. O achado foi ao acaso, em exames de rotina do criadouro, pois o animal não apresentava sinais clínicos decorrentes de parasitoses.

No ano de 2001 foi feito o primeiro registro de criptosporidiose em P. concolormantido em cativeiro na Argentina (14). Essa parasitose também já foi relatada em seres humanos, cães, gatos, bovinos, suínos, ovinos, equinos $(15,16)$ e felinos silvestres como Leopardus Weiidi e Felis concolor $(17,18)$.

A trichuríase observada nesse relato pode acarretar lesões nas mucosas cecais e gastrenterites em animais parasitados (19). Segundo a literatura, esse helminto na forma larval é responsável pela enfermidade conhecida como larva migrans visceral em humanos (20).

Neste estudo, não foi possível diferenciar morfologicamente entre T. gondii e Besnoitia sp. Sobre T. gondi, sabe-se que é um parasito comum entre os felinos, embora a maioria das pesquisas apresente apenas o diagnóstico sorológico. No Brasil, em uma pesquisa visando a avaliar a soroprevalência de Toxoplasma em pumas mantidos em cativeiro em 20 Estados observou-se uma positividade de 48\% (21). No presente estudo considera-se importante ressaltar a presença de oocistos nas fezes, o que demonstra que os pumas são disseminadores em potencial dessa zoonose e não apenas portadores. Entretanto, o gênero Besnoitia ainda não foi relatado 
em $P$. concolor, embora esse protozoário tenha sido observado em outros felinos (22).

Com base nos diagnósticos obtidos, conclui-se que o P. concolor é hospedeiro de Trichuris sp., Cryptosporidium sp. e Toxoplasma gondii/Besnoitiasp. Novas pesquisas devem ser realizadas, tanto para a atuação do homem em estratégias de preservação, quanto para verificar o real potencial dessa espécie como portadora e vinculadora de zoonoses.

\section{REFERÊNCIAS}

1. Anderson AE. A critical review of the literature on puma (Felis concolor). Division of Wildlife Colorado: Special Report; 1983.

2. Hansen K. Cougar the American lion. Flagstaff: Northland Publishing; 1992.

3. Machado ABM, Fonseca GAB, Machado RB, Aguiar LMS, Lins LV. Livro vermelho das espécies ameaçadas de extinção na fauna de Minas Gerais. Belo horizonte: Fundação biodiversities; 1998.

4. Barnes JH. Parasites. In: Harrison GJ, Harrison LR. Clinical avian medicine and surgery: including aviculture. Philadelphia: Saunders; 1986. p. 472-85.

5. Tavela A, Araujo JM, Braga FR, Silva AR, Araújo JV, Paula TAR. Identificação dos gêneros de helmintos em onça parda (Puma concolor) na zona da mata mineira. Biológico. 2007;69(2):113-98.

6. Patton S, Rabinowitz A, Randolph S, Johnson S. A coprological survey of parasites of wild Neotropical Felidae. J Parasitolol. 1986;72(4):517-20.

7. Greiner EC, Roelke ME, Atkinson CT, Dubey JP, Wright SD. Sarcocystis sp. in muscles of free-ranging Florida (USA) panthers and cougars (Felis concolor). J Wildl Dis. 1989;25(4):623-8.

8. Rickard LG, Foreyt WJ. Gastrointestinal parasites of cougars (Felis concolor) in Washington and the first report of Ollulanus tricuspis in a sylvatic felid from North America. J Wildl Dis. 1992;28(1):130-3.

9. Dunbar MR, McClaughlin GS, Murphy DM, Cunningham MW. Pathogenicity of the hookworm, Ancylostoma pluridentatum, in a Florida panther (Felis concolor coryi) kitten. J Wildl Dis. 1994;30(4):548-51.

10. Wehinger KA, Roelke ME, Greiner EC. Ixodid ticks from panthers and bobcats in Florida. J Wildl Dis. 1995;31(4):480-5.
11. Lamm MG, Roelke ME, Greiner EC, Steible CK. Microfilariae in the free-ranging Florida panther (Felis concolor coryı). J Wildl Dis. 1997;64(1):137-41

12. Aramini JJ, Stephen C, Dubey JP. Toxoplasma gondii in Vancouver Island cougars (Felis concolor vancouverensis): Serology and oocyst shedding. J Parasitol. 1998;84(2):438-40.

13. Hoffman RP. Diagnóstico de parasitismo veterinário. Porto Alegre: Sulina; 1987.

14. Pezzani BC, Batista LE, Apezteguia M, Basualdo JA. Prevalence of Cryptosporidium spp. in mammals of La Plata and its rural areas. Acta Bioq Clin Latinoamer. 2001;35(4):521-6.

15. Cubas ZS, Silva JCR, Catão-Dias JL. Tratado de animais silvestres. São Paulo: Roca; 2006. p. 1226-50.

16. Fayer R, Morgan U, Upton SJ. Epidemiology of Cryptosporidium: transmission, detection and identification. Int J Parasitol. 2000;30(12-13):1305-22.

17. Cabral DD, Barbosa FC, Strasser C, Barsotti SRH. Exame de fezes de mamíferos silvestres para verificação de parasitismo por Cryptosporidium sp. Bioscience J. 2001;17(1):77-83.

18. Belmonte CO, Soares JF, Da Silva AS, Silva MK, Salomão EL, Monteiro SG. Ocorrência de Giardia sp. e Cryptosporidium sp. em Leopardus weidii de vida livre. Ciência Rural. 2008;38(2):546-7.

19. Huber F, Bomfim TCB, Gomes RS. Comparação entre infecção por Cryptosporidium sp. e por Giardia sp. em gatos sob dois sistemas de criação. Rev Bras Parasitol Vet. 2002;11(1):7-12.

20. Sakano T, Hamamoto K, Kobayashi Y, Sakata Y, Tsuji M, Usiu T. Visceral larva migrans caused by Trichuris vulpis. Arch Dis Child. 1980;55(8):631-3.

21. Silva JCR, Ogassawara S, Adamia CH, Ferreira F, Gennari SM, Ferreira-Neto JS. Soroprevalence of Toxoplasma gondii in captive neotropical felids from Brazil.Vet Parasitolog. 2001;102(3):217-24.

22. Mckenna PB, Charleston WAG. Coccidia (Protozoa:Sporozoasida) of cats and dogs. III. The occurrence of a species of Besnoitia in cats. N Z Vet J. 1980;28(6):120-2.

Recebido: 05/06/2007

Received: 06/05/2007

Aprovado: 03/12/2007

Approved: $12 / 03 / 2007$ 